\title{
Computed tomography-based opportunistic osteoporosis assessment: a comparison of two software applications for lumbar vertebral volumetric bone mineral density measurements
}

\author{
Mischa Woisetschläger ${ }^{1,2} \wedge$, Martin Hägg ${ }^{1,2}$, Anna Spångeus ${ }^{3}$ \\ ${ }^{1}$ Department of Radiology and Department of Health, Medicine and Caring Sciences, Linköping University, Linköping, Sweden; ${ }^{2}$ Center for \\ Medical Image Science and Visualization (CMIV), Linköping University, Linköping, Sweden; ${ }^{3}$ Department of Acute Internal Medicine and \\ Geriatrics and Department of Health, Medicine and Caring Sciences, Linköping University, Linköping, Sweden
}

Correspondence to: Mischa Woisetschläger, MD, PhD. Department of Radiology, Building 411, level 11, University Hospital Linköping, 58185 Linköping, Sweden. Email: mischa.woisetschlager@liu.se.

Background: We aimed to compare two volumetric bone mineral density (vBMD) analysis programs, regarding (I) agreement of vBMD values based on mono- and dual-energy computed tomography (MECT and DECT) scans and (II) suitability for analyzing DECT data obtained at different energies.

Methods: We retrospectively analyzed two abdominal CT datasets: one performed in a MECT scan (vertebrae L1-L3) and one in a DECT scan (vertebrae L1-L4). Each dataset included different individuals [MECT 15 patients (45 vertebrae) and DECT 12 patients (48 vertebrae), respectively]. vBMD analysis was conducted using Philips IntelliSpace (IP) and Mindways qCT Pro (MW). Regarding the DECT scans, vBMD analysis was done at three different energies: 80, 150 and synthetic $120 \mathrm{kVp}$ and for MECT scan at $120 \mathrm{kVp}$. For comparison of vBMD results between different software (aim 1) MECT $120 \mathrm{kVp}$ and DECT synthetic $120 \mathrm{kV}$ data was used. For analyzing suitability of using different DECT energies for vBMD assessment (aim 2) all three DECT energies were used and results from each software was analyzed separately.

Results: vBMD assessed with MW and IP, respectively correlated significantly for both the MECT ( $\mathrm{r}=0.876$; $\mathrm{P}<0.001)$ and DECT $(\mathrm{r}=0.837 ; \mathrm{P}<0.001)$ scans, but the $v B M D$ values were lower in using IP for $v B M D$ assessment ( $8 \%$ and 14\% lower for MECT and DECT, respectively; $\mathrm{P}=0.001)$. Regarding the different DECT energies, using MW for vBMD assessment showed significant correlations in vBMD results between $120 \mathrm{kVp}$ and the two other energies $(\mathrm{r}=0.988$ and $\mathrm{r}=0.939)$ and no significant differences in absolute vBMD values $(\mathrm{P}>0.05)$. The IP analysis as well showed significant correlation between $120 \mathrm{kVp}$ and the other energies ( $\mathrm{r}=0.769$ and $\mathrm{r}=0.713$, respectively), but differences in absolute $\mathrm{vBMD}$ values between the energies $(\mathrm{P} \leq 0.001)$.

Conclusions: We show that the correlations between the vBMD derived from the two investigated software solutions were generally good but that absolute vBMD value did differ and might impact the clinical diagnosis of osteoporosis. Though small, our study data indicate that vBMD might be assessed in energies other than $120 \mathrm{kVp}$ when using MW but not when using IP.

Keywords: Osteoporosis; bone mineral density (BMD); dual-energy computed tomography (DECT); opportunistic; CT

Submitted Aug 27, 2020. Accepted for publication Oct 26, 2020.

doi: $10.21037 /$ qims-20-1013

View this article at: http://dx.doi.org/10.21037/qims-20-1013

$\wedge$ ORCID: 0000-0003-0066-4985. 


\section{Introduction}

Osteoporosis is a bone disorder characterized by low bone mineral density (BMD), which causes bone fragility, with increased risk of fracture even with low-energy trauma (1-4). These fractures are very common (i.e., occurring in a half of women and a fifth of men with low BMD) and are associated with high morbidity and a significant socioeconomic burden $(5,6)$. Furthermore, patients with a previous fracture have a higher risk of new fractures, potentially resulting in a fracture cascade, with imminent fracture risk (i.e., in the period soon after the initial fracture) being particularly increased (7). This highlights the need for fast and effective post-fracture screening to identify high-risk individuals in need of anti-osteoporotic treatment to prevent fractures. However, despite the availability of effective antiosteoporotic medications, there is a huge gap between the guidelines and reality, with a treatment gap estimated to be between $63-73 \%$ in Europe (8). In an attempt to overcome this issue, Fracture Liaison Services (FLSs) have been set up worldwide for more effective and reliable management of post-fracture patients.

The current gold standard for osteoporosis diagnosis is BMD measurement using the 2D method dual-energy X-ray absorptiometry (DXA). DXA is a well-recognized method with extensive longitudinal data. However, it has several methodological limitations including overestimation of spinal BMD with increasing age due to wrongly including arthritis and aortic calcification (which increase with age) in the BMD analysis $(2,3)$. Another possible limitation is that DXA machines are only used for BMD measurement, i.e., they necessitate an extra visit for the patient for BMD measurement only. From a clinical point of view, this extra visit in the FLS might be a logistic challenge in the clinical routine with an increased risk of loss to follow-up as well as extra costs associated with this dedicated procedure.

Various computed tomography (CT)-based methods can be used to potentially improve bone quality assessment and make the diagnosis process in FLSs more effective $(4,9,10)$. FLSs could perform opportunistic volumetric BMD (vBMD) assessment using CT scans conducted at the time of fracture occurrence (i.e., when detecting a vertebral fracture using abdominal or thoracic CT scans) as well as identifying high-risk individuals during opportunistic screening when investigating patients for other diagnoses (3,10-18). It is important that these CT-based methods are shown to be reliable and stable across various software programs, CT machines and scan protocols (i.e., not only protocols specific to skeletal imaging). In this way, $\mathrm{vBMD}$ measurements can be obtained without any additional radiation exposure or the need for dedicated clinical visits.

There are several vBMD analysis software programs on the market where analysis can be made without the need of an external calibration phantom in each scan. The technique used for estimating vBMD and calibration differs. Two of the software solutions available are Philips IntelliSpace (IP) and Mindways (MW). IP software uses single-energy CT datasets for $\mathrm{vBMD}$ analysis; it is a phantomless method that uses "internal vBMD calibration", with paraspinal muscle and fat tissues as reference materials. MW software uses single-energy datasets for $\mathrm{vBMD}$ analysis; this software further requires a dedicated phantom for $\mathrm{vBMD}$ calibration of the CT scanner (4) a procedure which is recommended to be performed once a month. Both techniques are recommended for MECT scan, but as DECT scans are becoming more prevalent it is of interest to investigate possible usage of these software in in-vivo DECT scan. Furthermore, a direct comparison of the $\mathrm{vBMD}$ derived from the different software would be clinically valuable as a possible difference could have an impact on clinical diagnosing of these patients.

Dual-energy CT (DECT) scans have been shown to gain adequate $\mathrm{vBMD}$ measurement in ex-vivo studies and in some in vivo-studies which also included investigation of analysis behavior in different contrast phases and synthetic non-contrast phases $(3,11-13,19-25)$ The latter is important as the usage of DECT scans in opportunistic osteoporosis screening will not always include a non-contrast scan. Another clinical prerequisite to be aware of is the usage of different energies and synthetic energies, i.e., preset mixture of the two energy sources, in the clinical routine care and a possibility that this might impact estimated $v B M D$ if DECT scans are used for opportunistic screening. More data are needed on in-vivo behavior of vBMD derived from DECT scans, including possible impact of the energies used. Ideally a direct comparison should be made to areal BMD (aBMD) derived from DXA scan, the gold standard for osteoporosis diagnostics However, in-vivo aBMD from DXA should be interpreted with care in elderly patients as confounding factors, such as fat, aortic calcification, spondyl arthritis, are wrongly included in the aBMD value. This might be an explanation why a direct comparison between DECT derived $\mathrm{vBMD}$ and DXA derived aBMD did show a poor correlation $(2,3,10)$ but was improved when adjusting DXA aBMD for aortic calcification and spondyl arthritis (3).

In this retrospective study, we aimed to compare two 


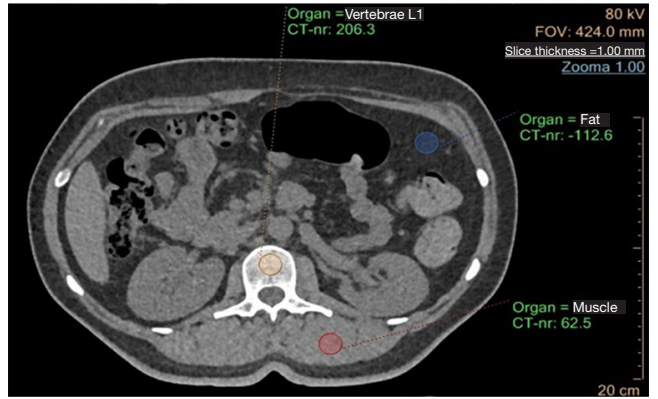

Figure 1 vBMD measurement of lumbar vertebra (yellow ROI) using Philips IntelliSpace (IP). This method is phantomless and uses internal calibration with HU values from paraspinal muscle tissue (red ROI) and fat tissue (blue ROI). vBMD, volumetric bone mineral density; ROI, region of interest.

commercially available vBMD analysis software programs (MW and IP) regarding: (I) agreement of vBMD values based on monoenergetic CT (MECT) and DECT scans and (II) suitability for analyzing DECT data obtained at different energies.

\section{Methods}

\section{Patients}

In the present study, we retrospectively analyzed two abdominal CT datasets: one performed in a MECT scan (vertebra L1-L3, total 45 vertebrae) and one in a DECT scan (vertebra L1-L4, total 48 vertebrae). Each dataset included different individuals.

From the DECT dataset, 12 patients ( 9 women; 3 men; mean age $69 \pm 9$ years; range, $47-84$ years) who underwent a clinically indicated abdominal DECT scan in 2016 were included. This dataset has previously been used in a study comparing DECT and DXA (3). From the MECT dataset, 15 patients ( 5 women; 10 men; mean age $70 \pm 8$ years; range, 55-86 years) with hepatocellular carcinoma scheduled for transarterial chemoembolization (TACE) from October 2016 to March 2019 were included.

\section{DECT scans and processing}

Patient scans in the DECT study were performed in a routine clinical setting using a 128 -section dual-source CT scanner (Somatom Force, Siemens Healthcare, Erlangen, Germany). The two X-ray tubes were operated at 80 and $150 \mathrm{kVp}$ with tin filter technology and a quality reference of $246 \mathrm{mAs}$ at $80 \mathrm{kVp}$ and $123 \mathrm{mAs}$ at $150 \mathrm{Sn} \mathrm{kVp}$. Pitch was
0.6. Images were reconstructed with an Admire $\mathrm{Br} 36 \mathrm{~d} / 3$ kernel with a section thickness of $1 \mathrm{~mm}$ and an increment of $0.5 \mathrm{~mm}$.

The patients were scanned in three phases (noncontrast, arterial and venous phases) but, for the present study, only the non-contrast scans were used. Based on these non-contrast scans, three datasets were analyzed, i.e., the two automatically generated 80 and $150 \mathrm{kVp}$ datasets, and a "synthetic $120 \mathrm{kVp}$ " dataset generated from postprocessing the dual-energy data using a 0.6 weighting factor for the $80 \mathrm{kVp}$ data and a 0.4 weighting factor for the $150 \mathrm{kVp}$ data (i.e., $60 \%$ from the $80 \mathrm{kVp}$ data and $40 \%$ from the $150 \mathrm{kVp}$ data).

\section{MECT scans}

Patient scans in the perfusion study were performed in a routine clinical setting using a 128-section dual-source CT scanner (Somatom Force, Siemens Healthcare, Erlangen, Germany) in single-energy mode. The scans were of the upper abdomen and they were performed without intravenous contrast agent. The scans were conducted at $120 \mathrm{kVp}$ with a reference value of $125 \mathrm{mAs}$.

\section{Radiation dose}

The DECT scans had a mean radiation dose [in doselength product (DLP)] of $413 \mathrm{mGycm}$ (230-630), and a mean effective radiation dose of $6.20 \mathrm{mSv}$ (3.4-9.45). The MECT scans had a mean radiation dose (in DLP) of 533 mGycm (306-808), and a mean effective radiation dose of $8.00 \mathrm{mSv}$ (4.59-12.12).

\section{BMD assessment}

For the vBMD analysis, the datasets were exported from our Picture Archiving System (PACS) to the two software programs: (I) IP (Bone mineral analysis, Philips, Amsterdam, the Netherlands) and (II) MW qCT PRO (version 2, Mindways, Austin, TX, USA).

Regarding the IP analysis, one region of interest (ROI) was manually placed in the trabecular bone in the middle portion of each lumbar vertebra on axial images (Figure 1). For each vertebra, two additional ROIs were placed in the fat and paraspinal muscle tissues, respectively, which served as reference materials for internal vBMD calibration. The ROI was placed in the middle trabecular part of the lumbar vertebrae. The thickness of the ROI was the same as the 


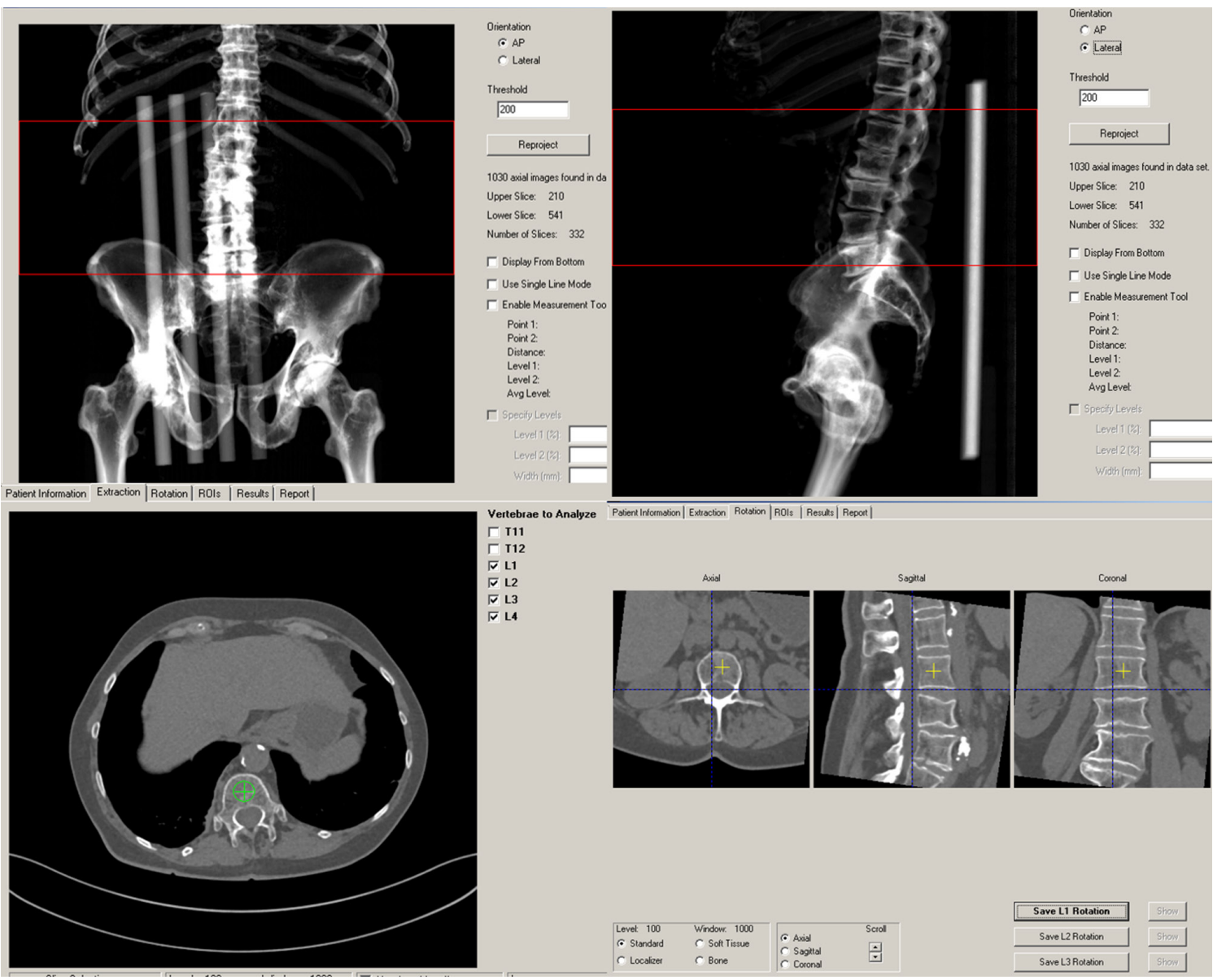

Figure 2 vBMD measurement using Mindways (MW) qCT Pro, with semi-automatic placement of ROIs in the trabecular part of the lumbar vertebrae. vBMD, volumetric bone mineral density; ROI, region of interest.

slice thickness, i.e., $1 \mathrm{~mm}$. The vBMD of the vertebra was then automatically calculated. The procedure was repeated for all vertebrae in the study.

In contrast to the IP analysis, the $M W$ analysis required calibration scans with Mindways' dedicated phantom prior to vBMD measurement. Calibrations were made once a month. No internal phantom during the clinical scans was then needed for the individual measurements. The MW software semi-automatically placed the ROIs in the trabecular part of the lumbar vertebrae (middle part), which could be manually adjusted before the vBMD values were calculated (Figure 2). The thickness of the ROI was $9 \mathrm{~mm}$.

In both studies, the BMD measurements were performed by two experienced radiologists (clinical radiology experience of 5-12 years).

\section{Impact on diagnosis}

In the clinical outcome analysis, for each patient, a mean vBMD was calculated for all the vertebrae assessed. In concordance with the World Health Organization guidelines (9), the clinical outcomes were defined as normal, osteopenic or osteoporotic based on vBMD values $>120$, $80-120,<80 \mathrm{~g} / \mathrm{cm}^{3}$, respectively.

\section{Statistical analysis}

SPSS Statistics (version 23.0, IBM Corp., Armonk, NY, USA) was used for the statistical analysis. The data were tested for normality with the Kolmogorov-Smirnov test and the Shapiro-Wilk test. The tests showed that the data were normally distributed. 

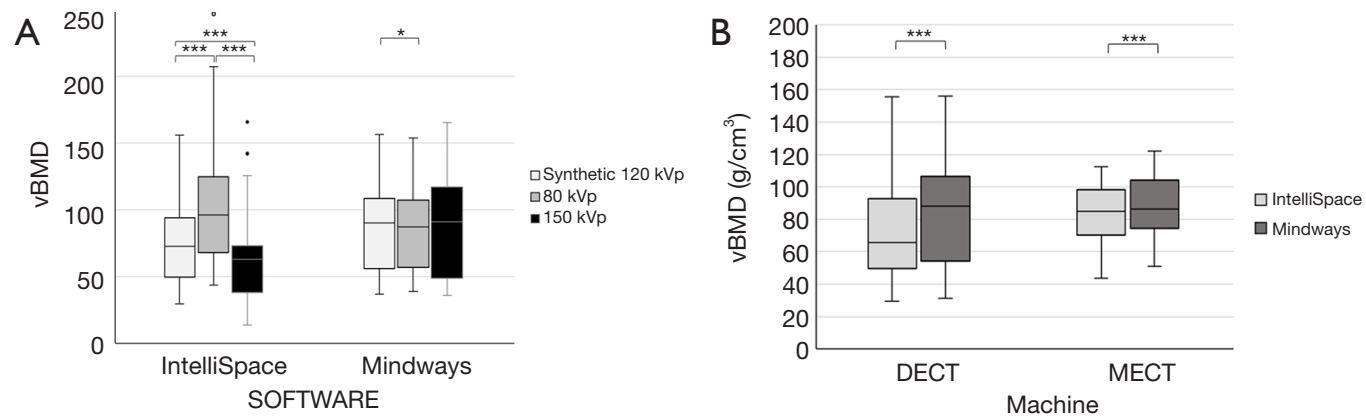

Figure 3 Effects on vBMD results from CT scans of different energies and CT techniques, using two different analysis software. (A) Mean vBMD values $\left(\mathrm{g} / \mathrm{cm}^{3}\right)$ and standard deviations using Philips IntelliSpace (IP) and Mindways (MW) for DECT data related to three $\mathrm{kVp}$ settings [80, 150 and synthetic $120 \mathrm{kVp}$ (60\%: $80 \mathrm{kV}$ data; 40\%: $150 \mathrm{kV}$ data)]. (B) Mean vBMD values (g/cm ${ }^{3}$ ) and standard deviations using IP and MW for MECT (120 kVp) and DECT (synthetic $120 \mathrm{kVp}$ ) data. *, $\mathrm{P}<0.05$; ${ }^{* *}, \mathrm{P}<0.001$. vBMD, volumetric bone mineral density; DECT, dual-energy computed tomography; MECT, monoenergetic computed tomography.

A parametric paired $t$-test was used for the comparison of vBMD values between two groups, and Pearson correlation analysis was used for the correlation analysis. For the comparison of the clinical outcomes between the two software programs, the $\mathrm{McNemar}$ test was used. A P value $<0.05$ was considered significant.

\section{Ethics}

The authors are accountable for all aspects of the work in ensuring that questions related to the accuracy or integrity of any part of the work are appropriately investigated and resolved. The trial was conducted in accordance with the Declaration of Helsinki (as revised in 2013). The study was approved by regional ethics/committee of the Faculty of Health Sciences, Linköping University, (2013/160-31 and 2014/63-32). An amendment for the MECT study was approved by the National Ethics Committee (2019-05855).

\section{Results}

\section{Comparisons of vBMD values based on DECT scans obtained at different energies}

There were significant correlations $(\mathrm{P}<0.001)$ in vBMD values between synthetic $120 \mathrm{kVp}$ and the two other energies when using the same analysis software, i.e., IP $(\mathrm{r}=0.769$ : synthetic 120 and $80 \mathrm{kVp} ; \mathrm{r}=0.713$ : synthetic 120 and $150 \mathrm{kVp})$ and $\mathrm{MW}(\mathrm{r}=0.988$ : synthetic 120 and $80 \mathrm{kVp}$; $\mathrm{r}=0.939$ : synthetic 120 and $150 \mathrm{kVp}$ ).

However, as shown in Figure $3 A$ and Table 1, when analyzing the DECT scans with IP software, the mean vBMD values differed significantly between the three energies; the mean vBMD was highest for $80 \mathrm{kVp}\left(100 \pm 44 \mathrm{~g} / \mathrm{cm}^{3}\right)$ followed by synthetic $120 \mathrm{kVp}\left(73 \pm 31 \mathrm{~g} / \mathrm{cm}^{3}\right)$ and $150 \mathrm{kVp}$ $\left(61 \pm 31 \mathrm{~g} / \mathrm{cm}^{3} ; \mathrm{P} \leq 0.001\right)$. More homogenous mean vBMD values were seen in the $\mathrm{MW}$ analysis $\left(84-88 \mathrm{~g} / \mathrm{cm}^{3} ; \mathrm{P}>0.05\right)$.

\section{Comparisons of vBMD values between IP and MW analyses using $120 \mathrm{kVp}$ data}

Regarding the DECT scans, there was a significant correlation in vBMD values between the MW and IP analyses involving synthetic $120 \mathrm{kVp}$ data $(\mathrm{r}=0.837$; $\mathrm{P}<0.001)$. However, the mean $v B M D$ value for synthetic $120 \mathrm{kVp}$ was significantly lower in the IP analysis $\left(73 \pm 31 \mathrm{~g} / \mathrm{cm}^{3}\right)$ than the $M W$ analysis $\left(88 \pm 35 \mathrm{~g} / \mathrm{cm}^{3}\right.$; $\mathrm{P}<0.001$ ) (Figure $3 B$ and Table 1). For these DECT data, the mean difference in $v B M D$ between the MW and IP analyses was $14 \%$ (Figure $4 A$ ).

Regarding the MECT scans, there was a significant correlation in vBMD values between the MW and IP analyses involving $120 \mathrm{kVp}$ data $(\mathrm{r}=0.876 ; \mathrm{P}<0.001)$. However, similar to the DECT result, the $\mathrm{vBMD}$ value for the MECT data was lower in the IP analysis $\left(81 \pm 19 \mathrm{~g} / \mathrm{cm}^{3}\right)$ than the MW analysis $\left(88 \pm 19 \mathrm{~g} / \mathrm{cm}^{3}\right.$; $\mathrm{P}<0.001$ ) (Figure $3 B$ and Table 1). For these MECT data, the mean difference in $v B M D$ between the MW and IP analyses was $8 \%$ (Figure $4 B$ ).

Regarding the pooled data (synthetic $120 \mathrm{kVp}$ data from DECT scans and $120 \mathrm{kVp}$ data from MECT scans), there was a significant correlation between the $\mathrm{MW}$ and IP analyses $(r=0.836 ; \mathrm{P}<0.001)$. Similar to the DECT and MECT results, the $\mathrm{vBMD}$ value for the pooled data 
Table 1 Volume bone mineral density in different energies and using different software

\begin{tabular}{|c|c|c|}
\hline Cohorts and energies & Philips IntelliSpace (IP) & Mindways (MW) \\
\hline \multicolumn{3}{|c|}{ MECT-cohort (45 vertebrae) } \\
\hline $120 \mathrm{kVp}$ & $81.3 \pm 19.5$ & $88.4 \pm 18.6$ \\
\hline \multicolumn{3}{|c|}{ DECT-cohort (48 vertebrae) } \\
\hline Synthetic $120 \mathrm{kVp}$ & $73.5 \pm 31.1$ & $87.8 \pm 34.9$ \\
\hline $150 \mathrm{kVp}$ & $61.3 \pm 31.1$ & $84.3 \pm 39.6$ \\
\hline
\end{tabular}

MECT, monoenergetic computed tomography; DECT, dual-energy computed tomography.

was lower in the IP analysis $\left(77 \pm 26 \mathrm{~g} / \mathrm{cm}^{3}\right)$ than the $\mathrm{MW}$ analysis $\left(88 \pm 28 \mathrm{~g} / \mathrm{cm}^{3} ; \mathrm{P}<0.001\right)$. For these pooled data, the mean difference in $\mathrm{vBMD}$ between the MW and IP analyses was $11 \%$ (Figure $4 C$ ).

\section{Clinical outcomes}

Regarding the diagnoses, the IP analysis identified $52 \%$ $(n=14)$ of the patients as osteoporotic, whereas the MW analysis identified slightly fewer patients as being in the osteoporotic range, i.e., $37 \%(\mathrm{n}=10 ; \mathrm{P}=0.082)$ (Figure 5).

\section{Discussion}

In the present study, we showed that opportunistic osteoporosis screening results based on analyzing abdominal CT scans (MECT and DECT scans) using commercially available software exhibited good correlation. However, the IP analysis generally showed significantly lower (8-14\%) mean vBMD values, which may influence diagnoses and subsequent clinical management. However, the study population was small, and thus results should be interpreted cautiously. Analysis of DECT scans obtained at different energies $(80,150$ and synthetic $120 \mathrm{kVp}$ ) showed that the vBMD values were significantly different in the IP analysis but not the MW analysis. This indicates that the IP analysis is not suitable for analyzing scans other than those obtained at $120 \mathrm{kVp}$.

Opportunistic vBMD measurement with a CT-based method, both in MECT and DECT mode, is useful, with the potential to contribute to an earlier discovery of osteoporosis without increased radiation exposure $(3,4,12,14-18)$. However, the BMD values determined by different software programs in the same patients across different machines should be comparable. The commonly used gold standard, the DXA scan, has been shown to differ by up to $11 \%$ (26) between the two most used machines, Hologic and Lunar. Our vBMD data determined using different software programs show a similar difference (8\%) regarding the MECT scans $(120 \mathrm{kVp})$ and a slightly higher difference (14\%) regarding the DECT scans (synthetic $120 \mathrm{kVp}$ ). In contrast to the DXA data, the present data were not obtained using specific protocols designed for skeletal imaging, tuned for optimum precision. Awareness of the differences between software programs is important, as different software programs might categorize patients differently (i.e., as having osteoporosis or not and as fulfilling specific treatment indications or not) and thus impact clinical management. One way of dealing with this problem would be by generating a conversion formula for use between the measurements from the two software programs, as already done for DXA measurements performed using either the Hologic or Lunar systems.

The reason for the different values between the software programs might be explained by the fact that the IP analysis only includes one ROI, with the thickness defined by the slice thickness of the CT examination. In contrast, MW is associated with an ROI depth of several millimeters (from 1-9 $\mathrm{mm}$ ), with a normal default of $9 \mathrm{~mm}$. This probably generates a more reliable measurement, as a larger volume of the trabecular bone of each vertebra is analyzed.

In comparison to DXA scans, there are several advantages related to conducting opportunistic vBMD measurements based on CT scans, including obtaining vBMD during clinical CT examinations without necessitating additional costs, time or radiation exposure $(1,3,14,17)$. Additionally, CT scans offer additional qualitative $3 \mathrm{D}$ information e.g., on the trabecular bone structure and finite element analysis; this eliminates the risk of overestimating BMD due to the presence of aortic calcification and arthritis (which are wrongly 
A
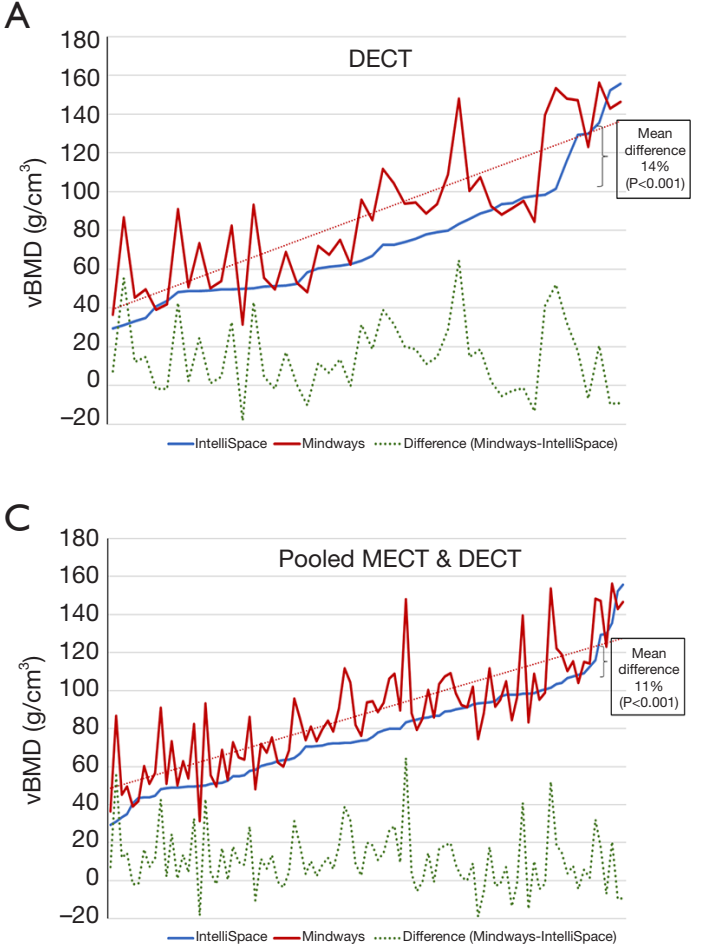

B

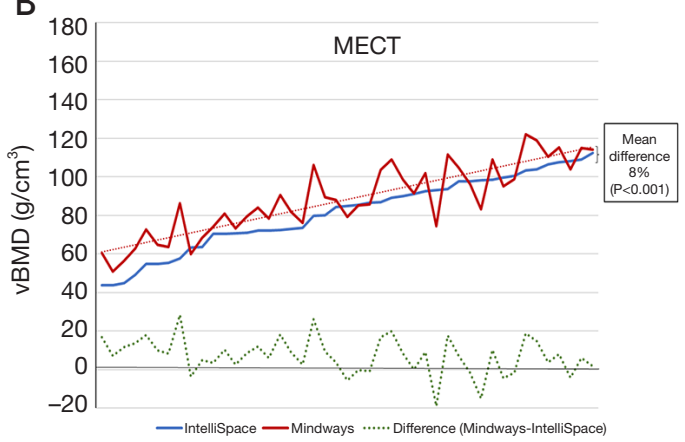

Figure 4 Correlation between vBMD results derived from Mindways (MW) and Philips IntelliSpace (IP) analysis software, respectively. (A) DECT data (synthetic $120 \mathrm{kVp}$ ), (B) MECT data $(120 \mathrm{kVp})$, (C) pooled MECT and DECT data: vBMD values $\left(\mathrm{g} / \mathrm{cm}^{3}\right)$ per vertebra in the IP analysis (blue line) and the MW analysis (red line), with a linear trendline for MW (dotted red line). Sorted by increasing IP vBMD values. The difference per vertebra (green line) is also shown. DECT data derived from synthetic $120 \mathrm{kVp}$ and MECT $120 \mathrm{kVp}$. vBMD, volumetric bone mineral density; DECT, dual-energy computed tomography; MECT, monoenergetic computed tomography.

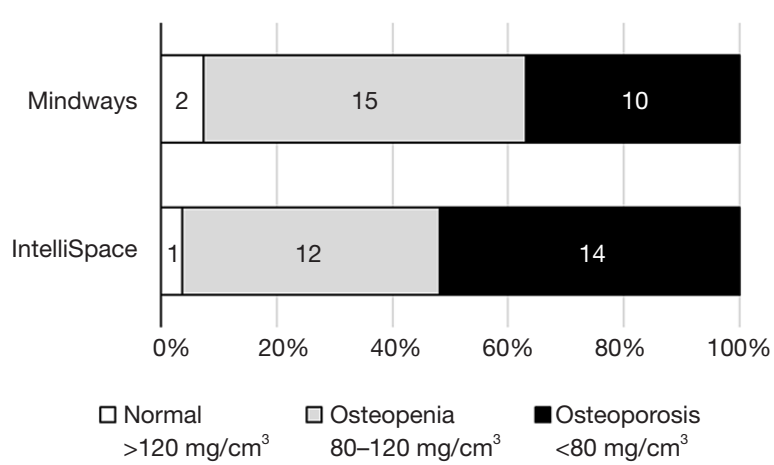

Figure 5 Distribution of patients in the normal (vBMD $>120 \mathrm{mg} / \mathrm{cm}^{3}$ ), osteopenia (vBMD $80-120 \mathrm{mg} / \mathrm{cm}^{3}$ ) and osteoporosis (vBMD $<80 \mathrm{mg} / \mathrm{cm}^{3}$ ) categories (in accordance with the World Health Organization criteria for quantitative CT) based on vBMD measurements determined by the Philips IntelliSpace (IP) and Mindways (MW) analyses $(\mathrm{P}=0.082)$. vBMD, volumetric bone mineral density; CT, computed tomography. included in the 2D BMD analysis involving DXA) (3). This might increase the precision of the diagnosis, the prediction of future fracture risk, and the appraisal of the benefits of preventive therapies.

In our vBMD analysis, we compared the $\mathrm{vBMD}$ values based on scans obtained at different energies and found a difference between the software programs. The IP analysis led to different $v B M D$ values for the same vertebrae depending on the $\mathrm{kVp}$ value of the scan, with $80 \mathrm{kVp}$ leading to the highest value and $150 \mathrm{kVp}$ the lowest. This might be explained by the fact that changes in $\mathrm{kVp}$ settings will affect $\mathrm{HU}$ values differently in different tissues; the more distant a tissue is from water $(\mathrm{HU}=0)$ in regard to $\mathrm{HU}$, the bigger the difference between measurements will become as the $\mathrm{kV}$ decreases. In addition, as the difference in the $\mathrm{kVp}$ value from the reference scan of $120 \mathrm{kVp}$ increases, the differences will rise exponentially the lower the $\mathrm{kVp}$ value becomes. It is important to note that IP software will 
not deliver robust $B M D$ values when used at $k V p$ values other than $120 \mathrm{kVp}$. This is becoming even more important as many examinations in a way to reduce radiation dose are undertaken with manually adjusted lower $\mathrm{kVp}$, e.g., 80, 90 or $100 \mathrm{kVp}$, or automatically adjusted $\mathrm{kVp}$-doses, e.g., in newer CT machines scans could be made with $\mathrm{kVp}$ adapting modes, which is a function that automatically selects the dose-optimized $\mathrm{X}$-ray tube voltage $(\mathrm{kVp})$ depending on the examination type. In contrast, the MW analysis led to consistent $\mathrm{BMD}$ values in abdominal scans at all $\mathrm{kVp}$ values tested.

Opportunistic osteoporosis screening should ideally be easily available and robust in various scan situations, both regarding energy as discussed above, and reason for making the CT scan.

Unlike IP with its internal calibration with patients own tissue (fat and muscle), MW needs monthly calibration with an external phantom scan. Good clinical routines for this is thus a prerequisite for using this software in a FLS. However, the fairly long intervals between calibration as well as the actual calibration time does not make these calibration step time-consuming for the organization. However, it requires some kind of osteoporosis calibration organization, which in some cases, especially when vBMD analysis is done irregularly could be an obstacle in adding opportunistic CT screening in a FLS. Regarding different scan situations many scans nowadays do not include noncontrast scans, but only different intravenous contrast phases. Previous studies have indicated that contrast interferes with $v B M D$ estimation and differs from noncontras scans. Virtual non-contrast scans from dual-energy examinations underestimate $\mathrm{vBMD}$ and therefore cannot be used for vBMD assessment. Least differences were seen using venous phase scans and adjusted venous phase imaging might be used for opportunistic BMD screening (22).

Limitations of the study include the small number of patients included and the use of only one vBMD measurement per method. Another limitation is the fact that the study consists of two different study groups. However, this made it possible to compare synthetic $120 \mathrm{kVp}$ data to real monoenergetic $120 \mathrm{kVp}$ data.

We cannot rule out selection bias of the data included and this in some way could affect the results. Thus, the MECT group consisted of a certain patient group (TACE treated patients). In the MECT group were more men than women in total $(10 v s$. 5) which does not reflect the distribution of patients with osteoporosis. But as all patients were compared intra-personally (within the same investigation) we believe the effect of this should not be of any major importance. We used dual-source CT for the DECT scans. There are other DECT techniques which possibly could give different results. Furthermore, we only investigated three different energies and with our data we cannot judge if other energies would produce similar vBMD.

\section{Conclusions}

Opportunistic vBMD measurement of lumbar vertebrae based on abdominal CT scans is feasible and could improve the diagnosis of patients with osteoporosis. However, the present study identified important differences between the software programs to be aware of. Although the correlation between the $\mathrm{vBMD}$ values from the two software programs was good, IP generally led to lower values than $M W$ (8-14\%), which could impact clinical management. Further studies are needed and a tool for converting between the measurements from different software programs should be created. Analyzing data related to energies other than $120 \mathrm{kVp}$ is feasible in MW analyses but not in IP analyses.

\section{Acknowledgments}

Funding: None.

\section{Footnote}

Conflicts of Interest: All authors have completed the ICMJE uniform disclosure form (available at http://dx.doi. org/10.21037/qims-20-1013). The authors have no conflicts of interest to declare.

Ethical Statement: The study was conducted in accordance with the Declaration of Helsinki (as revised in 2013). The study was approved by regional ethics/committee of the Faculty of Health Sciences, Linköping University (2013/160-31 and 2014/63-32). An amendment for the MECT study was approved by the National Ethics Committee (2019-05855). Informed consent was obtained from all participants.

Open Access Statement: This is an Open Access article distributed in accordance with the Creative Commons Attribution-NonCommercial-NoDerivs 4.0 International License (CC BY-NC-ND 4.0), which permits the noncommercial replication and distribution of the article with 
the strict proviso that no changes or edits are made and the original work is properly cited (including links to both the formal publication through the relevant DOI and the license). See: https://creativecommons.org/licenses/by-nc-nd/4.0/.

\section{References}

1. Engelke K, Adams JE, Armbrecht G, Augat P, Bogado CE, Bouxsein ML, Felsenberg D, Ito M, Prevrhal S, Hans DB, Lewiecki EM. Clinical use of quantitative computed tomography and peripheral quantitative computed tomography in the management of osteoporosis in adults: the 2007 ISCD Official Positions. J Clin Densitom 2008;11:123-62.

2. Bolotin HH. DXA in vivo BMD methodology: an erroneous and misleading research and clinical gauge of bone mineral status, bone fragility, and bone remodelling. Bone 2007;41:138-54.

3. Woisetschläger $M$, Spångeus A. Model for improved correlation of BMD values between abdominal routine Dual energy CT data and DXA scans. Eur J Radiol 2018;99:76-81.

4. Brown JK, Timm W, Bodeen G, Chason A, Perry M, Vernacchia F, DeJournett R. Asynchronously calibrated quantitative bone densitometry. J Clin Densitom 2017;20:216-25.

5. Jonsson E, Eriksson D, Åkesson K, Ljunggren Ö, Salomonsson S, Borgström F, Ström O. Swedish osteoporosis care. Arch Osteoporos 2015;10:222.

6. Hernlund E, Svedbom A, Ivergård M, Compston J, Cooper C, Stenmark J, McCloskey EV, Jönsson B, Kanis JA. Osteoporosis in the European Union: medical management, epidemiology and economic burden. A report prepared in collaboration with the International Osteoporosis Foundation (IOF) and the European Federation of Pharmaceutical Industry Associations (EFPIA). Arch Osteoporos 2013;8:136.

7. Toth E, Banefelt J, Åkesson K, Spångeus A, Ortsäter G, Libanati C. History of previous fracture and imminent fracture risk in Swedish women aged 55 to 90 years presenting with a fragility fracture. J Bone Miner Res 2020;35:861-8.

8. Borgström F, Karlsson L, Ortsäter G, Norton N, Halbout P, Cooper C, Lorentzon M, McCloskey EV, Harvey NC, Javaid MK, Kanis JA; International Osteoporosis Foundation. Fragility fractures in Europe: burden, management and opportunities. Arch Osteoporos 2020;15:59.
9. The American College of Radiology. Acr-Spr-Ssr practice parameter for the performance of musculoskeletal quantitative computed tomography (QCT). Available online: https://www.acr.org/-/media/ACR/Files/PracticeParameters/QCT.pdf

10. Hofmann P, Sedlmair M, Krauss B, Wichmann JL, Bauer RW, Flohr TG, Mahnken AH. Phantom-less bone mineral density (BMD) measurement using dual energy computed tomography-based 3-material decomposition. In: Medical Imaging 2016: Computer-Aided Diagnosis, 2016;9785:97853E.

11. Wesarg S, Kirschner M, Becker M, Erdt M, Kafchitsas K, Khan MF. Dual-energy CT-based assessment of the trabecular bone in vertebrae. Methods Inf Med 2012;51:398-405.

12. Wesarg S, Kafchitsas K, Erdt M, Khan MF. CAD of osteoporosis in vertebrae using dual-energy CT. In: Perth: 2010 IEEE 23rd International Symposium on ComputerBased Medical Systems (CBMS), 2010:358-63.

13. Booz C, Hofmann PC, Sedlmair M, Flohr TG, Schmidt B, D'Angelo T, Martin SS, Lenga L, Leithner D, Vogl TJ, Wichmann JL. Evaluation of bone mineral density of the lumbar spine using a novel phantomless dual-energy CT post-processing algorithm in comparison with dual-energy X-ray absorptiometry. Eur Radiol Exp 2017;1:11.

14. Michalski AS, Besler BA, Michalak GJ, Boyd SK. CTbased internal density calibration for opportunistic skeletal assessment using abdominal CT scans. Med Eng Phys 2020;78:55-63.

15. Pan Y, Shi D, Wang H, Chen T, Cui D, Cheng X, Lu Y. Automatic opportunistic osteoporosis screening using lowdose chest computed tomography scans obtained for lung cancer screening. Eur Radiol 2020;30:4107-16.

16. Keaveny TM, Clarke BL, Cosman F, Orwoll ES, Siris ES, Khosla S, Bouxsein ML. Biomechanical computed tomography analysis (BCT) for clinical assessment of osteoporosis. Osteoporos Int 2020;31:1025-48.

17. Ziemlewicz TJ, Binkley N, Pickhardt PJ. Opportunistic osteoporosis screening: addition of quantitative CT bone mineral density evaluation to CT colonography. J Am Coll Radiol 2015;12:1036-41.

18. Summers RM, Baecher N, Yao J, Liu J, Pickhardt PJ, Choi JR, Hill S. Feasibility of simultaneous computed tomographic colonography and fully automated bone mineral densitometry in a single examination. J Comput Assist Tomogr 2011;35:212-6.

19. Wichmann JL, Booz C, Wesarg S, Kafchitsas K, Bauer RW, Kerl JM, Lehnert T, Vogl TJ, Khan MF. Dual-energy 
CT-based phantomless in vivo three-dimensional bone mineral density assessment of the lumbar spine. Radiology 2014;271:778-84.

20. Qin L, Huang J, Yu P, Yan J, Ge Y, Lu Y, Yan F, Wang L, Du L. Accuracy, agreement, and reliability of DECTderived vBMD measurements: an initial ex vivo study. Eur Radiol 2021;31:191-9.

21. Cataño Jimenez S, Saldarriaga S, Chaput CD, Giambini $H$. Dual-energy estimates of volumetric bone mineral densities in the lumbar spine using quantitative computed tomography better correlate with fracture properties when compared to single-energy BMD outcomes. Bone 2020;130:115100.

22. Zopfs D, Lennartz S, Zaeske C, Merkt M, Laukamp KR, Reimer RP, Maintz D, Borggrefe J, Grosse Hokamp N. Phantomless assessment of volumetric bone mineral density using virtual non-contrast images from spectral detector computed tomography. Br J Radiol 2020;93:20190992.

23. Laugerette A, Schwaiger BJ, Brown K, Frerking LC, Kopp
FK, Mei K, Sellerer T, Kirschke J, Baum T, Gersing AS, Pfeiffer D, Fingerle AA, Rummeny EJ, Proksa R, Noël PB, Pfeiffer F. DXA-equivalent quantification of bone mineral density using dual-layer spectral CT scout scans. Eur Radiol 2019;29:4624-34.

24. van Hamersvelt RW, Schilham AMR, Engelke K, den Harder AM, de Keizer B, Verhaar HJ, Leiner T, de Jong PA, Willemink MJ. Accuracy of bone mineral density quantification using dual-layer spectral detector CT: a phantom study. Eur Radiol 2017;27:4351-9.

25. Van Hedent S, Su KH, Jordan DW, Eck B, Liang F, Kessner R, Kuo JW, Buls N, Klahr P, Ros P, Muzic RF Jr. Improving bone mineral density assessment using spectral detector CT. J Clin Densitom 2019;22:374-81.

26. Genant HK, Grampp S, Glüer CC, Faulkner KG, Jergas M, Engelke K, Hagiwara S, Van Kuijk C. Universal standardization for dual $\mathrm{x}$-ray absorptiometry: patient and phantom cross-calibration results. J Bone Miner Res 1994;9:1503-14.

Cite this article as: Woisetschläger $M$, Hägg $M$, Spångeus A. Computed tomography-based opportunistic osteoporosis assessment: a comparison of two software applications for lumbar vertebral volumetric bone mineral density measurements. Quant Imaging Med Surg 2021;11(4):13331342. doi: 10.21037/qims-20-1013 